\title{
Neoadjuvant docetaxel with or without carboplatin plus dual HER2 blockade for HER2-positive breast cancer: a retrospective multi- center Chinese study
}

\author{
Minhao Lv ${ }^{1}$, Huihui Guo ${ }^{2}$, Chao Wang ${ }^{3}$, Peiqi $\operatorname{Tian}^{1}$, Youzhao Ma ${ }^{1}$, Xiuchun Chen ${ }^{1}$, Suxia Luo ${ }^{4}$ \\ ${ }^{1}$ Department of Breast Disease, The Affiliated Cancer Hospital of Zhengzhou University \& Henan Cancer Hospital, Zhengzhou, China; \\ ${ }^{2}$ Department of General Surgery, The Jiyuan People's Hospital, Jiyuan, China; ${ }^{3}$ Department of General Surgery, The Huaxian People's Hospital, \\ Huaxian, China; ${ }^{4}$ Department of Medical Oncology, The Affiliated Cancer Hospital of Zhengzhou University \& Henan Cancer Hospital, \\ Zhengzhou, China \\ Contributions: (I) Conception and design: X Chen, S Luo; (II) Administrative support: S Luo; (III) Provision of study materials or patients: All authors; \\ (IV) Collection and assembly of data: M Lv, Y Ma, P Tian, H Guo, C Wang; (V) Data analysis and interpretation: M Lv; (VI) Manuscript writing: All \\ authors; (VII) Final approval of manuscript: All authors. \\ Correspondence to: Suxia Luo. Department of Medical Oncology, The Affiliated Cancer Hospital of Zhengzhou University \& Henan Cancer Hospital, \\ No. 127, Dongming Road, Zhengzhou 450008, China. Email: zlyyluosuxia0361@zzu.edu.cn.
}

Background: This study aimed to compare the real-world efficacy and safety of the TCbHP regimen (docetaxel, carboplatin, trastuzumab and pertuzumab) and the THP regimen (docetaxel, trastuzumab and pertuzumab) as neoadjuvant therapy for Chinese patients with human epidermal growth factor receptor 2 (HER2)-positive breast cancer.

Methods: We compared efficacy and safety outcomes from 72 Chinese patients with HER2-positive breast cancer who underwent neoadjuvant dual HER2 blockade plus TCb or T chemotherapy and surgery between March 2019 and June 2020.

Results: All 72 patients were women (32-76 years old) and the overall pathological complete response (pCR) rate was $70.8 \%(51 / 72)$. The pCR rates were $76.1 \%(35 / 46)$ for the TCbHP regimen and $61.5 \%$ $(16 / 26)$ for the THP regimen $(\mathrm{P}=0.28)$. Univariate analyses revealed that $\mathrm{pCR}$ was associated with clinical T classification $(\mathrm{P}=0.024)$, AJCC stage $(\mathrm{P}=0.042)$, estrogen receptor $(\mathrm{ER})$ status $(\mathrm{P}=0.002)$, progesterone receptor $(\mathrm{PR})$ status $(\mathrm{P}=0.035), \mathrm{Ki}-67$ index $(\mathrm{P}<0.001)$, and immunohistochemical HER2 status $(\mathrm{P}<0.001)$. Multivariate analyses revealed that pCR was independently predicted by ER status (OR: 0.227, 95\% CI: 0.053-0.852; $\mathrm{P}=0.032$ ) and immunohistochemical HER2 status (OR: 43.673, 95\% CI: 6.801-875.86; $\mathrm{P}<0.001$ ). The common adverse events for both regimens included neutropenia, anemia, thrombocytopenia, nausea, and diarrhea. Relative to the THP group, the TCbHP group had higher frequencies of grade 3-4 thrombocytopenia $(17 \%$ vs. $0 \%, \mathrm{P}=0.044)$ and grade $3-4$ diarrhea $(15 \%$ vs. $0 \%, \mathrm{P}=0.044)$. Both regimens had very good cardiac safety.

Conclusions: These results suggest that both TCbHP and THP regimens may be useful neoadjuvant treatments for high-risk early or locally advanced HER2-positive breast cancer. Both regimens had generally good safety outcomes, although clinicians should be aware of the risks of grade 3-4 thrombocytopenia and diarrhea during TCbHP treatment. Elderly patients who require neoadjuvant therapy may benefit from 6 cycles of THP treatment, based on its good efficacy and mild adverse events.

Keywords: Breast cancer; neoadjuvant chemotherapy; pathological complete response; safety

Submitted Sep 27, 2020. Accepted for publication Dec 04, 2020.

doi: 10.21037/gs-20-791

View this article at: http://dx.doi.org/10.21037/gs-20-791 


\section{Introduction}

Breast cancer is the most common malignant tumor in women, and overexpression of human epidermal growth factor receptor 2 (HER2) is present in $20-25 \%$ of breast cancer patients (1). The HER2-positive molecular subtype is aggressive, and whilst historically associated with a poor prognosis, outcomes have greatly improved since the introduction of monoclonal antibodies that target HER2 (e.g., trastuzumab and pertuzumab) (2).

Neoadjuvant chemotherapy has become a standard of care for locally advanced breast cancer and is increasingly being used for the treatment of early breast cancer $(3,4)$. This is because the prognosis of patients with pathological complete response (pCR) after neoadjuvant chemotherapy is significantly better than that of patients without pCR (5). Many studies have indicated that neoadjuvant dual HER2 blockade using pertuzumab and trastuzumab, plus chemotherapy, provides better outcomes than single HER2 blockade using trastuzumab plus chemotherapy. This has made dual HER2 blockade plus chemotherapy the standard neoadjuvant strategy for high-risk HER2positive breast cancer $(6,7)$ Moreover, the TRYPHAENA and TRAIN-2 studies revealed no significant difference in pCR after dual HER2 blockade with or without anthracycline $(8,9)$. Dual HER2 blockade plus nonanthracycline chemotherapy may, therefore, be a useful neoadjuvant strategy for high-risk HER2-positive breast cancer. Carboplatin is another traditional cytotoxic drug that is used in neoadjuvant therapy, however this is known to produce various toxic effects, such as febrile neutropenia, anemia, and thrombocytopenia (10). The ideal neoadjuvant strategy should, therefore, maximize therapeutic benefits and minimize toxic effects. This retrospective study aimed to evaluate the efficacy and safety of neoadjuvant therapy using docetaxel with or without carboplatin, plus trastuzumab and pertuzumab, in Chinese patients with HER2-positive breast cancer.

We present the following article in accordance with the STROBE reporting checklist (available at http://dx.doi. org/10.21037/gs-20-791).

\section{Methods}

\section{Patients}

This multi-center retrospective study evaluated data from The Affiliated Cancer Hospital of Zhengzhou University, The Jiyuan People's Hospital, and The Huaxian People's
Hospital. We searched the medical records of breast cancer patients who received neoadjuvant therapy and underwent surgery at the three hospitals between March 2019 and June 2020. All patients fulfilled the following criteria: (I) ECOG score of $0-1$; (II) histopathological confirmation of HER2positive invasive breast cancer; (III) central confirmation of positive HER2 status via immunohistochemistry (IHC 3+) or fluorescence in situ hybridization (FISH, mandatory for IHC $2+$ tumors); (IV) breast cancer that was judged to be operable (T1-3N1M0), locally advanced (T2-3N2-3M0; T4a-c, any $\mathrm{N}, \mathrm{M} 0$ ), or inflammatory (T4d, any $\mathrm{N}, \mathrm{M} 0$ ); (V) neoadjuvant treatment using the TCbHP or THP regimens; (VI) complete treatment and follow-up data; and (VII) a baseline left ventricular ejection fraction (LVEF) of $\geq 55 \%$.

All procedures performed in this study involving human participants were in accordance with the Declaration of Helsinki (as revised in 2013). The retrospective protocol of the study was approved by the ethics committee of The Affiliated Cancer Hospital of Zhengzhou University. All patients provided informed consent for use of their data for research purposes.

\section{Pathological and immunohistochemical analysis}

At baseline, the primary tumor and suspected axillary or clavicular lymph nodes were pathologically diagnosed via ultrasound-guided core needle biopsy. Tumor status for estrogen receptor (ER), progesterone receptor (PR), HER2, and $\mathrm{Ki}-67$ were determined. Positive expressions of ER and $\mathrm{PR}$ were identified based on positive staining for $\geq 1 \%$ of the nuclei, while positive staining for $<1 \%$ of the nuclei was considered a negative result. The HER2 status was considered positive based on an immunohistochemical score of $3+$ or $2+$ with FISH confirmation.

\section{Treatments}

The choice of neoadjuvant regimen and its dosage was determined by the physician in charge based on the patient's condition and disease. The neoadjuvant therapy regimens were the TCbHP regimen (docetaxel, carboplatin, trastuzumab, and pertuzumab) and the THP regimen (docetaxel, trastuzumab, and pertuzumab). The recommended TCbHP regimen used docetaxel $\left(75 \mathrm{mg} / \mathrm{m}^{2}\right)$ and carboplatin [area under the concentration-time curve (AUC): $6 \mathrm{mg} / \mathrm{mL} / \mathrm{min}$ ] intravenously, once every 3 weeks. The recommended THP regimen used docetaxel (75- 
$100 \mathrm{mg} / \mathrm{m}^{2}$ ) intravenously, once every 3 weeks. The trastuzumab loading dose was $8 \mathrm{mg} / \mathrm{kg}$, which was followed by $6 \mathrm{mg} / \mathrm{kg}$ intravenously, once every 3 weeks. The pertuzumab loading dose was $840 \mathrm{mg}$, which was followed by $420 \mathrm{mg}$ intravenously, once every 3 weeks. Patients who experienced severe drug reactions during chemotherapy had their doses modified accordingly. All patients underwent surgery after 6 cycles of neoadjuvant therapy. Because T-DM1 (trastuzumab emtansine) is not currently available in China, trastuzumab and pertuzumab continued to be given after surgery during the study period, regardless of whether pCR was achieved.

Before the first cycle of neoadjuvant therapy, patients underwent various evaluations to identify any distant metastasis through the use of mammography, breast and abdominal ultrasonography, chest and head computed tomography, bone scan, breast magnetic resonance imaging (MRI), and blood tests. Patients routinely received pegylated recombinant human granulocyte colony stimulating factor during the neoadjuvant chemotherapy. General adverse reactions, such as thrombocytopenia and diarrhea, need to be treated with symptomatic support. Intolerable adverse drug reactions were initially managed by reducing the chemotherapy dose by $20-25 \%$. However, the chemotherapy drug was stopped if reactions persisted after two consecutive dose reductions.

All patients had pathologically confirmed axillary lymph node metastasis at baseline, completed 6 cycles of neoadjuvant chemotherapy, then underwent axillary lymph node dissection and breast surgery. Complete pathological evaluations were performed for the breast and axillary specimens. All patients also underwent postoperative radiotherapy and 1 year of dual HER2 blockade using trastuzumab and pertuzumab. None of the patients received adjuvant chemotherapy, regardless of pCR status, although postoperative endocrine therapy was provided to patients with ER-positive and/or PR-positive breast cancers.

\section{Evaluations}

Tumor responses were assessed every 1-2 cycles via ultrasonography or MRI, and were categorized as complete response (CR), partial response (PR), stable disease (SD), and progressive disease (PD), based on the Response Evaluation Criteria in Solid Tumors. We defined pCR as the absence of invasive carcinoma in the breast and axillary lymph nodes. Adverse reactions were observed and recorded during treatment and were evaluated according to version
4.0 of the Common Terminology Criteria for Adverse Events. Cardiac ultrasonography was used to monitor changes in LVEF, which was evaluated at baseline, at every cycle of neoadjuvant treatment, and every 3 months during adjuvant dual HER2 blockade. The disease-free survival (DFS) interval was calculated from the date of the primary surgery to the first instance of relapse, death, or the followup cut-off (July 1, 2020).

\section{Statistical analysis}

Continuous variables were evaluated using the $t$-test or Wilcoxon rank sum test. Categorical variables were evaluated using the chi-squared test or Fisher's exact test. Univariate analyses were performed to identify relevant risk factors ( $\mathrm{P}$ value $<0.05$ ), which were incorporated into a multivariate logistic stepwise regression model. The optimal model was identified based on the minimum Akaike information criterion. All statistical analyses were performed using R software (version 3.6.3) and differences were considered statistically significant at $P$ values of $<0.05$.

\section{Results}

\section{Patient characteristics}

The study included 72 patients who were treated between March 30, 2019 and June 10, 2020 at the three centers. Patients received either the TCbHP regimen (46 patients) or the THP regimen (26 patients), and their clinicopathological features are shown in Table 1. The mean age was 53.0 years (range: $32-76$ years), and evaluations revealed ER positivity in $41.7 \%$ of cases (30/72), PR positivity in $37.5 \%$ of cases $(27 / 72)$, and a Ki67 index of $\geq 30 \%$ in $83.3 \%$ of cases $(60 / 72)$. The clinical T classification was cT1 (12 patients), cT2 (43 patients), cT3 (14 patients), and cT4 (3 patients). The clinical N classification was cN1 (18 patients), cN2 (24 patients), and cN3 (30 patients). Comparison of the TCbHP and THP groups revealed significant differences in age $(\mathrm{P}<0.001)$ and menstrual status $(\mathrm{P}<0.001)$. However, no significant differences were observed in the EGOC score $(\mathrm{P}=0.063)$, clinical $\mathrm{T}$ classification $(\mathrm{P}=0.358)$, clinical $\mathrm{N}$ classification $(\mathrm{P}=0.063)$, AJCC stage $(\mathrm{P}=0.136)$, ER status $(\mathrm{P}=0.623)$, $\mathrm{PR}$ status $(\mathrm{P}=0.314)$, immunohistochemical HER2 status $(\mathrm{P}=1.0)$, Ki-67 index $(\mathrm{P}=0.517)$, RECIST-based treatment response $(\mathrm{P}=0.891)$, or surgical methods $(\mathrm{P}=1.0)$ (Table 1). 
Table 1 Baseline characteristics

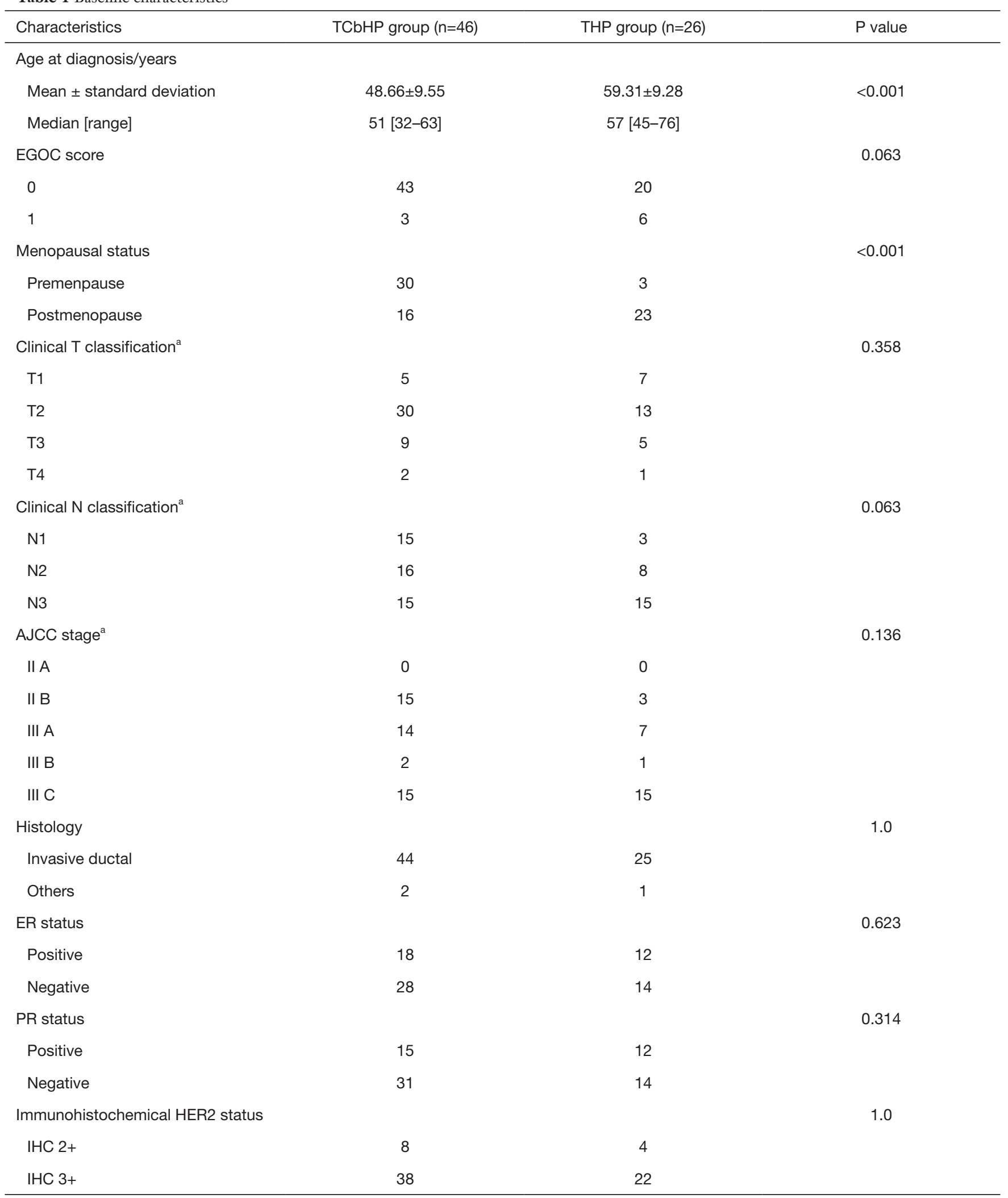

Table 1 (continued) 
Table 1 (continued)

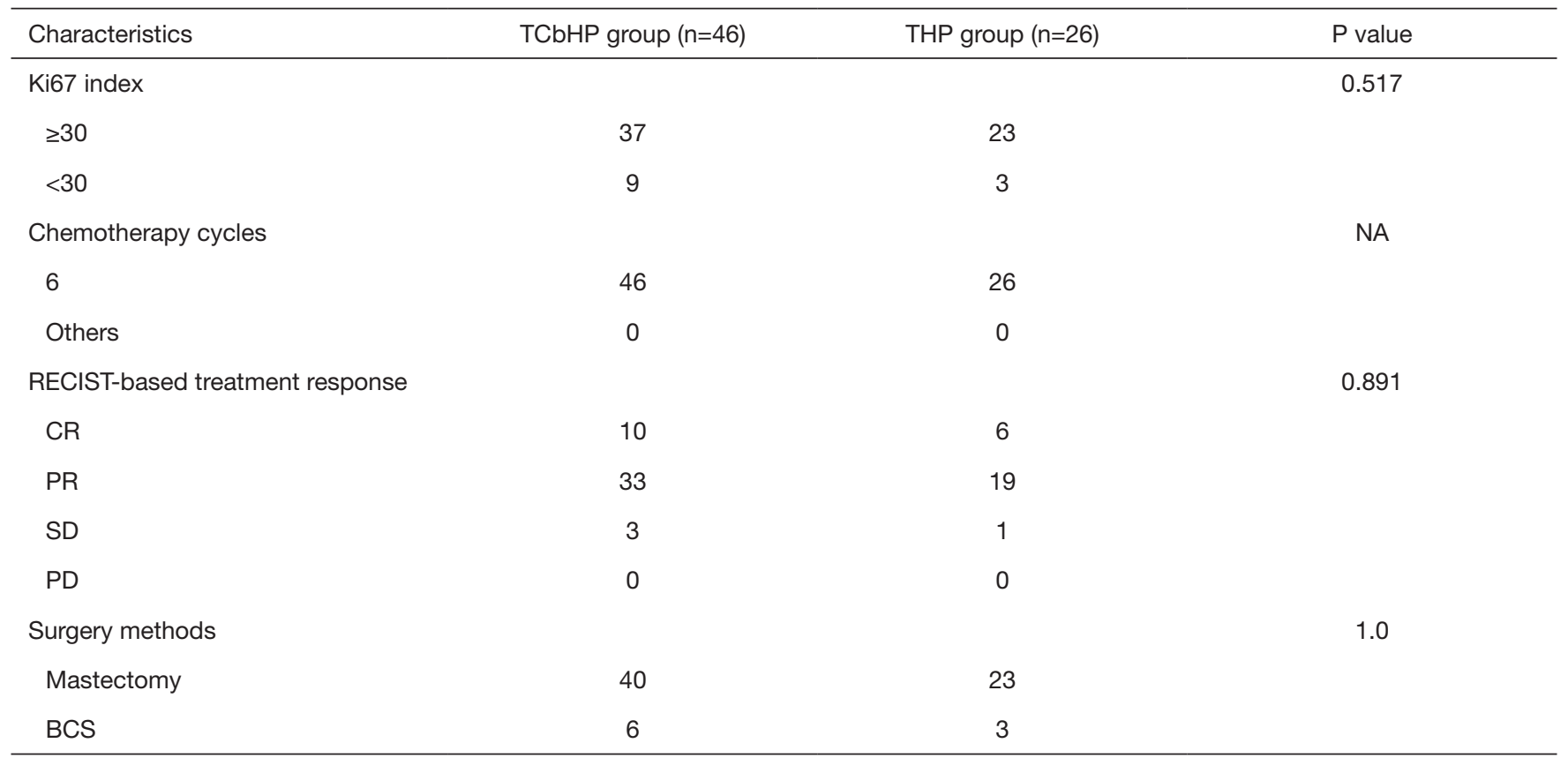

${ }^{a}$ Clinical tumor size and nodal status were assessed according to the 8th edition of the American Joint Committee on Cancer TNM staging system. ECOG, Eastern Cooperative Oncology Group; CR, complete response; PR, partial response; SD, stable disease; PD, progressive disease; BCS, breast-conserving surgery.

\section{Treatment responses}

All 72 patients completed 6 cycles of neoadjuvant therapy, which included dual HER2 blockade plus chemotherapy. None of the patients had PD, four had SD, and the remaining patients had PR or CR. There were no significant differences between the TCbHP and THP groups in terms of the CR rate $[21.7 \%(10 / 46)$ vs. $23.1 \%(6 / 26)]$, the PR rate $[71.7 \%(33 / 46) v s .73 .1 \%(19 / 26)]$, or the SD rate $[6.5 \%$ (3/46) vs. 3.8\% (1/26)] $(\mathrm{P}=0.891)$ (Table 1). Furthermore, there was no significant difference in the mean maximum reduction in the target lesion's size from baseline (TCbHP: $-70.25 \% \pm 27.19 \%$ vs. THP: $-72.47 \% \pm 21.20 \%, \mathrm{P}=0.953$ ) (Figure 1).

After surgery, pCR (ypT0/is ypN0) was achieved for $51 / 72$ patients $(70.8 \%)$, with pCR rates of $76.1 \%(35 / 46)$ in the TCbHP group and $61.5 \%(16 / 26)$ in the THP group $(\mathrm{P}=0.28)$ (Table 2 and Figure 2). The univariate analyses revealed that $\mathrm{pCR}$ was significantly associated with the clinical $\mathrm{T}$ classification $(\mathrm{P}=0.024)$, AJCC stage $(\mathrm{P}=0.042)$, ER status $(\mathrm{P}=0.002)$, PR status $(\mathrm{P}=0.035)$, Ki67 index $(\mathrm{P}<0.001)$, and immunohistochemical HER2 status $(\mathrm{P}<0.001)$. The multivariate analyses revealed that pCR was independently predicted by ER status (odds ratio:
0.227, 95\% confidence interval: $0.053-0.852 ; \mathrm{P}=0.032)$ and immunohistochemical HER2 status (odds ratio: 43.673, 95\% confidence interval: 6.801-875.86; $\mathrm{P}<0.001)$.

All patients had complete follow-up data with a median duration of 8 months (range: 1-15 months). One patient in the TCbHP group experienced chest wall recurrence at 3 months after surgery, and is currently receiving secondline anti-HER2 therapy. None of the remaining patients experienced local recurrence or distant metastasis.

\section{Toxicity and safety}

The 72 patients frequently received modified initial doses of the chemotherapy drugs. In the TCbHP group, the initial dosages of docetaxel were $76-80 \mathrm{mg} / \mathrm{m}^{2}(13 / 46,28 \%)$ and $70-75 \mathrm{mg} / \mathrm{m}^{2}(33 / 46,72 \%$ ) (Figure $3 A$ ) and the initial dosages of carboplatin were $5-6 \mathrm{mg} / \mathrm{mL} / \mathrm{min}(26 / 46,57 \%)$, 4-5 $\mathrm{mg} / \mathrm{mL} / \mathrm{min}(14 / 46,30 \%)$, and $3-4 \mathrm{mg} / \mathrm{mL} / \mathrm{min}(6 / 46$, $13 \%$ ) (Figure $3 B$ ). In the THP group, the initial dosages of docetaxel were $91-100 \mathrm{mg} / \mathrm{m}^{2}$ (5/26, 19\%), $81-90 \mathrm{mg} / \mathrm{m}^{2}$ (9/26, 35\%), $76-80 \mathrm{mg} / \mathrm{m}^{2}(6 / 26,23 \%)$, and $70-75 \mathrm{mg} / \mathrm{m}^{2}$ (6/26, 23\%) (Figure 3C).

Most common adverse events were considered grade 

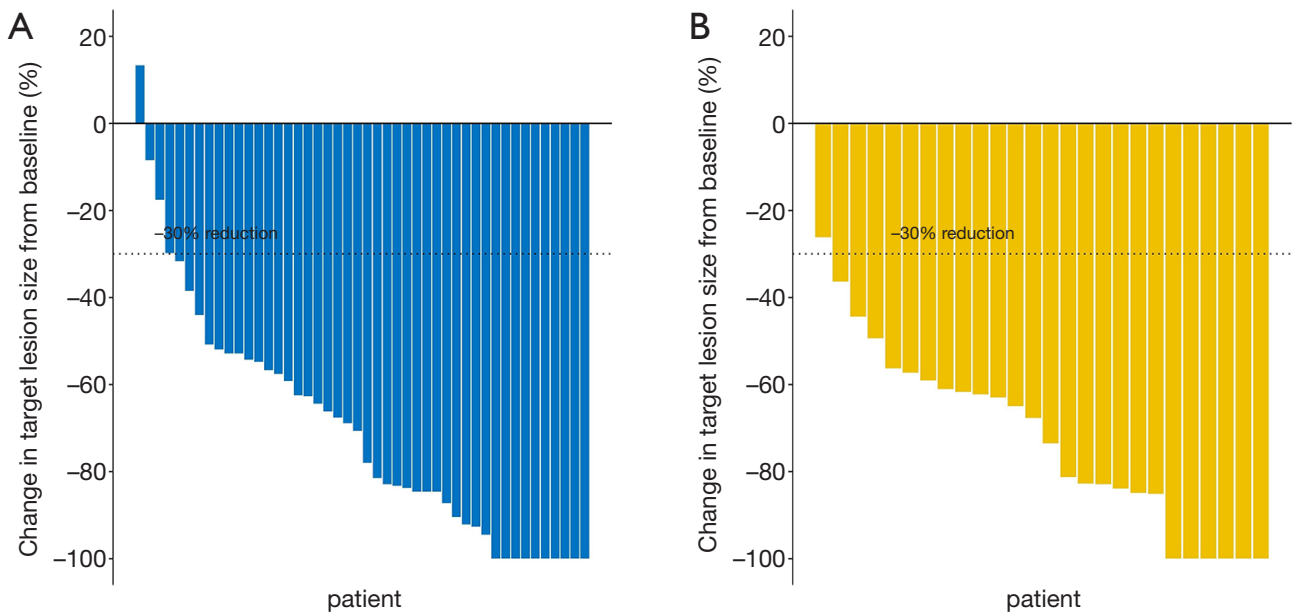

Figure 1 The maximum reduction in target lesion size from baseline during neoadjuvant therapy using (A) pertuzumab, trastuzumab, and docetaxel plus carboplatin (TCbHP) or (B) pertuzumab and trastuzumab plus docetaxel (THP). There was no statistical difference in the mean maximum reduction in the target lesion's size between the TCbHP and THP groups $(-70.25 \% \pm 27.19 \% v s$. $-72.47 \% \pm 21.20 \%$, $\mathrm{P}=0.953)$.

1-2, and included neutropenia, anemia, thrombocytopenia, alanine aminotransferase increased, nausea, vomiting, diarrhea, and rash (Table 3). The most common grade 3-4 adverse events were neutropenia, anemia, thrombocytopenia, nausea, and diarrhea (Table 3). Relative to the THP group, the TCbHP group had higher frequencies of grade $3-4$ thrombocytopenia (17\% vs. $0 \%$, $\mathrm{P}=0.044)$ and grade $3-4$ diarrhea $(15 \%$ vs. $0 \%, \mathrm{P}=0.044)$ (Table 3). Figure 4 shows that there was no significant difference in the mean maximum LVEF changes from baseline in the TCbHP and THP groups $(-4.30 \% \pm 3.12 \%$ vs. $-5.62 \% \pm 2.26 \%, \mathrm{P}=0.206)$. Only one patient in the THP group experienced a LVEF decline of $\geq 10 \%$ from baseline during the neoadjuvant period. That patient had a history of coronary heart disease and symptomatic treatment helped normalize the LVEF, which allowed them to continue receiving treatment.

In the TCbHP group, $37 \%$ of patients (17/46) had a carboplatin dose reduction and $13 \%$ of patients $(6 / 46)$ required carboplatin discontinuation because of intolerable toxicity. However, docetaxel doses in the TCbHP group did not need to be adjusted (Table 3). In the THP group, $12 \%$ of patients $(3 / 26)$ had a docetaxel dose reduction and no patient needed to discontinue docetaxel. In the TCbHP group, the proportions of cases with a carboplatin dose reduction were $53.85 \%(14 / 26)$ at an initial dose of 5$6 \mathrm{mg} / \mathrm{mL} / \mathrm{min}, 21.43 \%(3 / 14)$ at an initial dose of $4-$ $5 \mathrm{mg} / \mathrm{mL} / \mathrm{min}$, and $0.0 \%(0 / 6)$ at an initial dose of $3-$
$4 \mathrm{mg} / \mathrm{mL} / \mathrm{min}$. Only patients with an initial dose of $5-$ $6 \mathrm{mg} / \mathrm{mL} / \mathrm{min}$ needed to stop using carboplatin $(6 / 26$, $23.08 \%)$ (Figure 5).

\section{Discussion}

Although HER2-positive breast cancer was originally associated with a poor prognosis, substantial improvements have been observed after the introduction of anti-HER2 therapy $(2,11,12)$. Neoadjuvant therapy is increasingly used in early breast cancer, especially for more invasive subtypes, such as HER2 positive subtypes. Neoadjuvant therapy can facilitate surgical treatment, determine drug sensitivity, and identify patients who do not achieve pCR and should receive intensive postoperative treatment $(13,14)$. The current standard neoadjuvant/adjuvant therapy for patients with high-risk early or locally advanced HER2positive breast cancer should involve dual HER2 blockade (trastuzumab and pertuzumab) and chemotherapy $(6,7,15)$.

The National Comprehensive Cancer Network Guide currently recommends AC-THP and TCbHP as neoadjuvant/adjuvant strategies, and the treatment strategy of AC-T plus one or two anti-HER2 drugs is supported by the most evidence. The APHINITY study evaluated 2,400 patients who received dual targeted therapy and found no significant difference in the 3-year invasive DFS rate between non-anthracycline chemotherapy (docetaxel + carboplatin) and anthracycline plus paclitaxel/docetaxel (15). 
Table 2 Univariate and multivariate analysis of factors associated with tpCR

\begin{tabular}{|c|c|c|c|c|c|}
\hline \multirow{2}{*}{ Characteristics } & \multicolumn{2}{|c|}{ Pathological evaluation } & \multirow{2}{*}{$\frac{\text { Univariate analysis }}{\mathrm{P} \text { value }}$} & \multicolumn{2}{|c|}{ Multivariate analysis } \\
\hline & $\operatorname{tpCR}(n=51)$ & Non-tpCR $(n=21)$ & & OR $(95 \% \mathrm{Cl})$ & $P$ value \\
\hline Age at diagnosis (years) & & & 0.737 & & \\
\hline$\leq 40$ & $8(66.7 \%)$ & $4(33.3 \%)$ & & & \\
\hline$>40$ & $43(71.7 \%)$ & $17(28.3 \%)$ & & & \\
\hline Clinical T classification & & & 0.024 & & \\
\hline $\mathrm{T} 2$ & $31(72.1 \%)$ & $12(27.9 \%)$ & & & \\
\hline T3 & $9(64.3 \%)$ & $5(35.7 \%)$ & & & \\
\hline $\mathrm{T} 4$ & $0(0.0 \%)$ & $3(100.0 \%)$ & & & \\
\hline Clinical N classification & & & 0.655 & & \\
\hline N3 & $23(76.7 \%)$ & $7(23.3 \%)$ & & & \\
\hline AJCC stage & & & 0.042 & & \\
\hline II B & $12(66.7 \%)$ & $6(33.3 \%)$ & & & \\
\hline III A & $16(76.2 \%)$ & $5(23.8 \%)$ & & & \\
\hline III B & $0(0.00 \%)$ & $3(100.0 \%)$ & & & \\
\hline III C & $23(76.7 \%)$ & $7(23.3 \%)$ & & & \\
\hline \multicolumn{2}{|c|}{ RECIST-based treatment response } & & 0.178 & & \\
\hline CR & $14(87.5 \%)$ & $2(12.5 \%)$ & & & \\
\hline PR status & & & 0.035 & & \\
\hline Positive & $15(55.6 \%)$ & $12(44.4 \%)$ & & & \\
\hline Negative & $36(80.0 \%)$ & $9(20.0 \%)$ & & & \\
\hline \multicolumn{2}{|c|}{ Immunohistochemical HER2 status } & & $<0.001$ & & \\
\hline IHC 2+ & $1(8.3 \%)$ & $11(91.7 \%)$ & & & \\
\hline IHC 3+ & $50(83.3 \%)$ & $10(16.7 \%)$ & & $43.673(6.801-875.86)$ & $<0.001$ \\
\hline Ki67 index & & & $<0.001$ & & \\
\hline$\geq 30$ & $48(80.0 \%)$ & $12(20.0 \%)$ & & & \\
\hline$<30$ & $3(25.0 \%)$ & $9(75.0 \%)$ & & & \\
\hline Chemotherapy regimen & & & 0.28 & & \\
\hline TCbHP & $35(76.1 \%)$ & $11(23.9 \%)$ & & & \\
\hline THP & $16(61.5 \%)$ & $10(38.5 \%)$ & & & \\
\hline
\end{tabular}

pCR, pathological complete response; tpCR, total pCR (ypTO/is ypN0); CR, complete response; PR, partial response; SD, stable disease; $\mathrm{IHC}$, immunohistochemical. 
Furthermore, the TRYPHAENA and TRAIN-2 studies revealed no significant difference in the pCR rates for neoadjuvant dual HER2 blockade with or without anthracycline treatment $(8,9)$, and the pCR rates were $>65 \%$ for all regimens that did not include anthracycline. In this context, neoadjuvant therapy selection should balance the expected therapeutic effect and potential adverse events. Cardiotoxicity is associated with the use of anthracyclines, trastuzumab, and pertuzumab, and the combination of these three drugs as neoadjuvant therapy carries a significantly

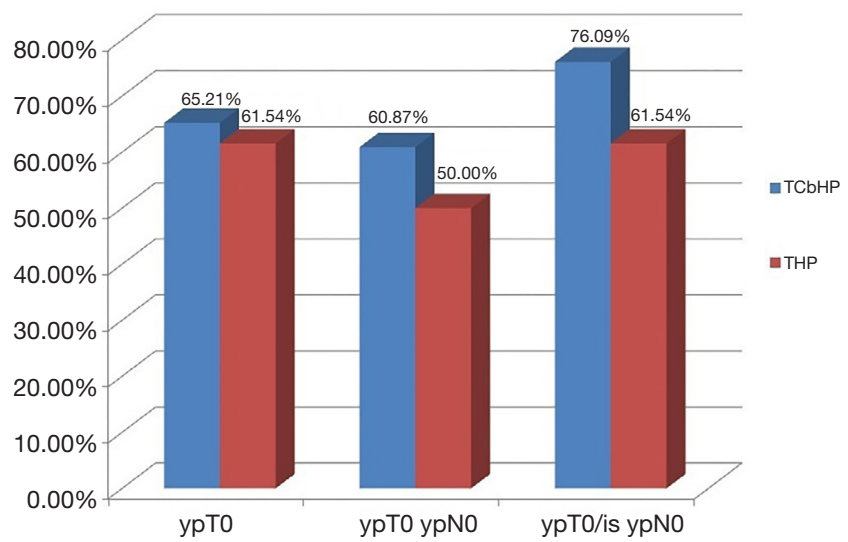

Figure 2 Comparison of the pathological complete response (pCR) between the TCbHP and THP groups. ypT0, no invasive carcinoma or carcinoma in situ remained in the breast lesion; ypT0 ypN0, no invasive carcinoma or carcinoma in situ remained in the breast lesion and axillary lymph nodes; ypT0/is ypN0, no invasive carcinoma remained in the breast lesion and axillary lymph nodes. increased risk of cardiotoxicity (15-17). Therefore, dual HER2 blockade combined with non-anthracycline chemotherapy may be a useful neoadjuvant strategy for high-risk HER2-positive breast cancer.

Many clinical studies have explored the efficacy and safety of the TCbHP regimen as neoadjuvant/adjuvant therapy $(8-10,15)$. The NeoSphere and PEONY studies have also indicated that 4 cycles of the THP regimen as neoadjuvant therapy was associated with high pCR rates $(6,7)$, although those studies also involved 3 postoperative cycles of the FEC regimen (fluorouracil, epirubicin and cyclophosphamide). To the best of our knowledge, ours is the first study to directly compare the efficacy and safety of the TCbHP and THP regimens as neoadjuvant therapy for HER2-positive breast cancer, and both regimens were delivered in 6 preoperative cycles with no postoperative chemotherapy.

Our study found that the pCR rate for the TCbHP group was $76.1 \%$, despite the high proportion of patients with $\mathrm{cN} 2-3$ disease (67.4\%), although others have indicated slightly lower pCR rates (56-66\%) (8-10). There are several possible explanations for higher pCR rate of TCbHP regimen in this study. The small sample size may have caused selection bias and/or the proportion of ER-negative patients was high in the TCbHP group (28/46, 60.9\%). The TRAIN-2 study revealed a pCR rate of up to $89 \%$ among ER-negative patients who received dual HER2targeted therapy plus chemotherapy (9), and our study also revealed a similar pCR rate among ER-negative patients (36/42, 85.7\%). For the THP regimen, the pCR rates were

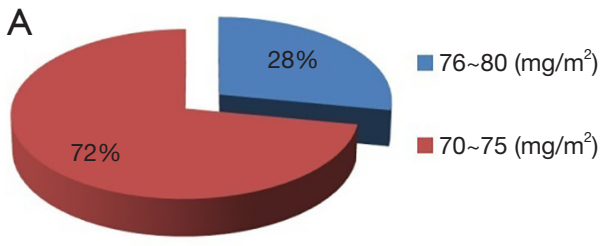

B
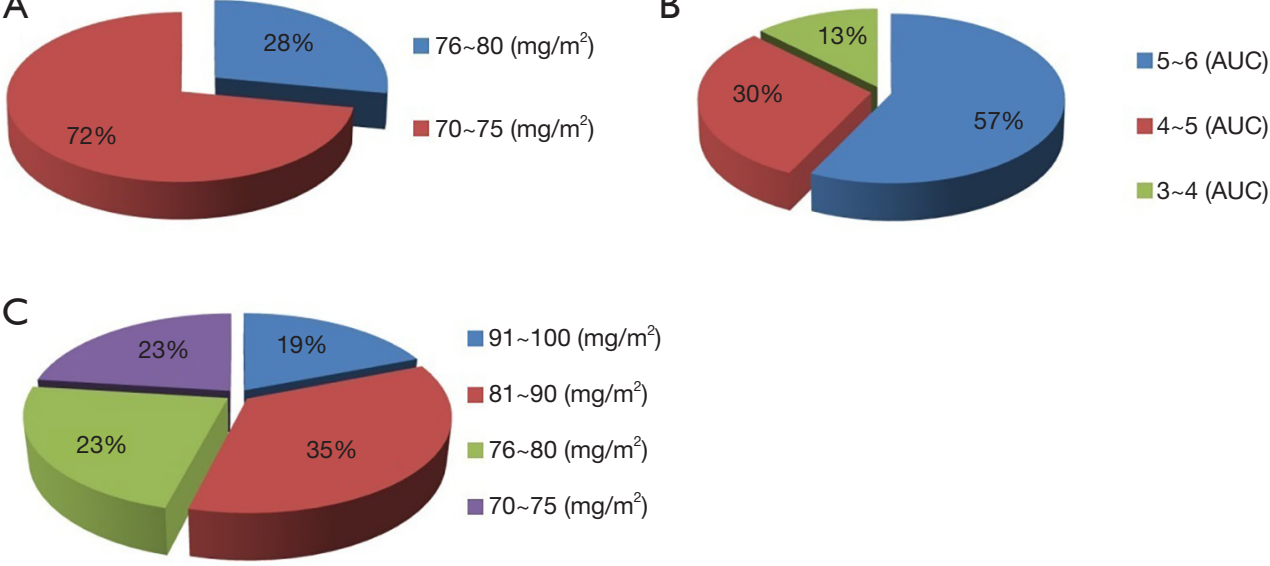

Figure 3 The initial dose distributions of the chemotherapy drugs in the TCbHP and THP groups. The initial dose distributions of (A) docetaxel in the TCbHP group, (B) carboplatin in the TCbHP group, and (C) docetaxel in the THP group. 
Table 3 Comparison of common adverse events

\begin{tabular}{|c|c|c|c|c|c|c|}
\hline AEs & \multicolumn{2}{|c|}{ TCbHP group $(n=46)$} & \multicolumn{2}{|c|}{ THP group $(n=26)$} & \multicolumn{2}{|c|}{$P$ value } \\
\hline Neutropenia & $31(67 \%)$ & $8(17 \%)$ & $15(58 \%)$ & $3(12 \%)$ & 0.451 & 0.735 \\
\hline Anaemia & $21(46 \%)$ & $6(13 \%)$ & $6(23 \%)$ & 0 & 0.077 & 0.081 \\
\hline Thrombocytopenia & $19(41 \%)$ & $8(17 \%)$ & $3(12 \%)$ & 0 & 0.009 & 0.044 \\
\hline Vomiting & $21(46 \%)$ & $3(7 \%)$ & $9(35 \%)$ & $1(4 \%)$ & 0.458 & 1.0 \\
\hline Diarrhea & $25(54 \%)$ & $7(15 \%)$ & $8(31 \%)$ & 0 & 0.084 & 0.044 \\
\hline Alanine aminotransferase increased & $9(20 \%)$ & $2(4 \%)$ & $3(12 \%)$ & 0 & 0.517 & 0.532 \\
\hline Rash & $6(13 \%)$ & 0 & $2(8 \%)$ & 0 & 0.702 & NA \\
\hline $\begin{array}{l}\text { Significant LVEF decline events }(\geq 10 \text { percentage } \\
\text { points from baseline and to }<50 \%)\end{array}$ & 0 & NA & $1(4 \%)$ & NA & 0.361 & NA \\
\hline Carboplatin dose reduction & $17(37 \%)$ & NA & NA & NA & & \\
\hline Carboplatin discontinuation & $6(13 \%)$ & NA & NA & NA & & \\
\hline Docetaxel dose reduction & 0 & NA & $3(12 \%)$ & NA & & \\
\hline Docetaxel discontinuation & 0 & NA & 0 & NA & & \\
\hline
\end{tabular}

AEs, adverse events; LVEF, left ventricular ejection fraction; NA, no available.
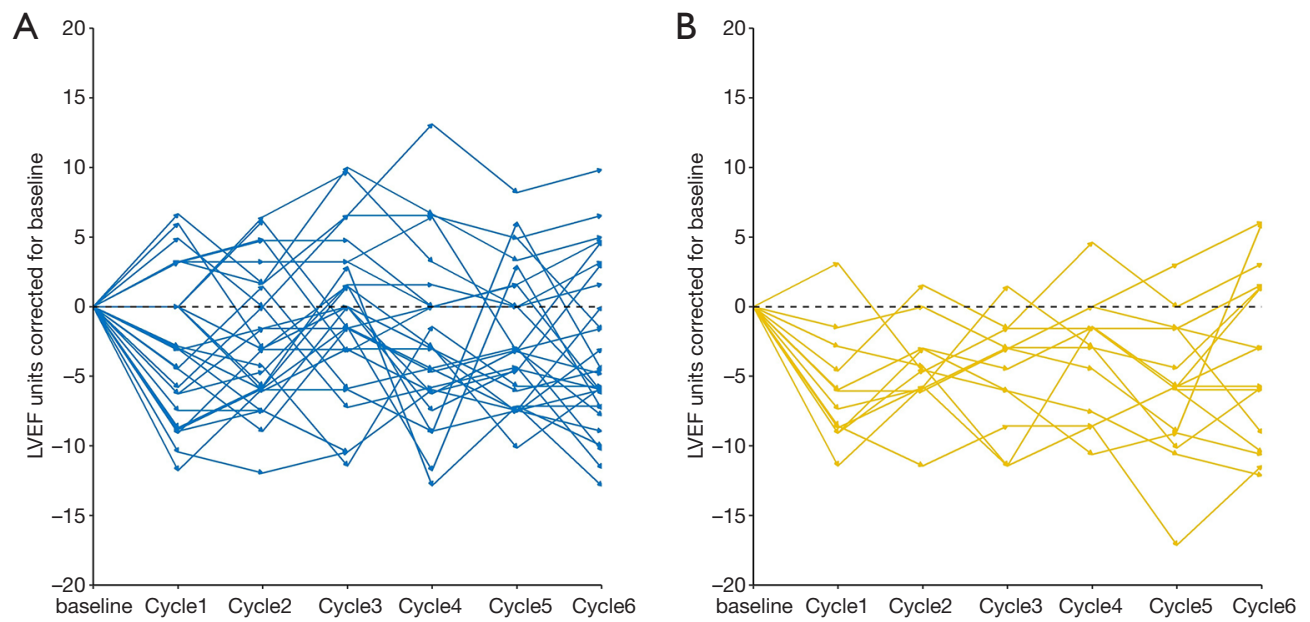

Figure 4 The relative LVEF changes from baseline in the groups that received (A) pertuzumab, trastuzumab, and docetaxel plus carboplatin (TCbHP) or (B) pertuzumab and trastuzumab plus docetaxel (THP). There was no statistical difference in the mean maximum relative decline in LVEF between the TCbHP and THP groups $(-4.30 \pm 3.12 v s .-5.62 \pm 2.26, \mathrm{P}=0.206)$. LVEF, left ventricular ejection fraction.

$45.8 \%$ in the NeoSphere study and $39.3 \%$ in the PEONY study $(6,7)$, while our study revealed a pCR rate of $61.5 \%$ (16/26). Selection bias related to the small sample size may also explain that difference, although it is also important to note that the NeoSphere and PEONY studies used 4 cycles of neoadjuvant therapy, while our study involved 6 cycles. 


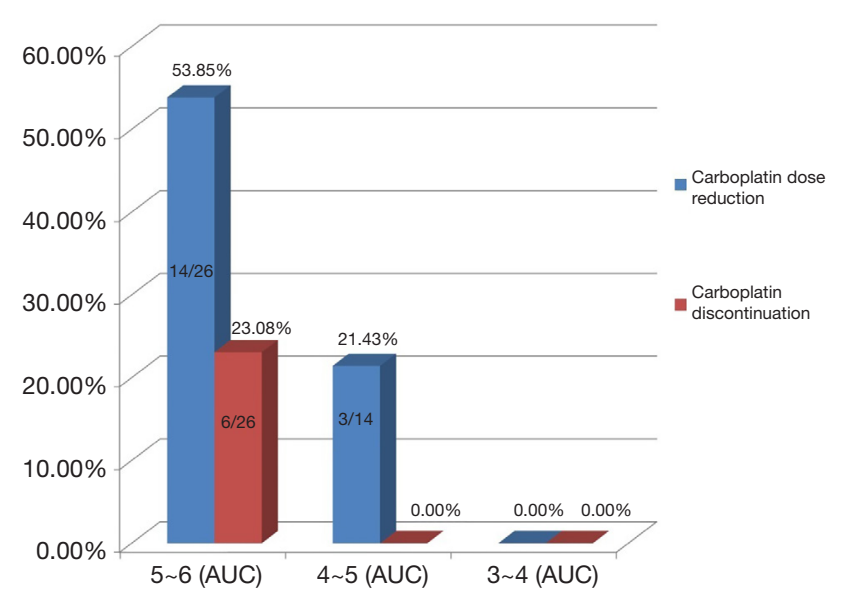

Figure 5 Comparison of dose reduction and discontinuation rates at different initial doses of carboplatin in the TCbHP group. AUC, area under the concentration-time curve.

Prolonging neoadjuvant treatment may, therefore, increase the $\mathrm{pCR}$ rate.

Our study found that, in real-world clinical practice, the TCbHP regimen tends to be selected for younger patients with good physical condition while the THP regimen tends to be selected for older and more frail patients. These results suggest that patient tolerance is an important consideration when choosing an appropriate treatment, and that elderly patients with potential complications and poor physical condition should receive a less toxic regimen. Thus, the imbalance in baseline characteristics between the TCbHP and THP groups was largely related to clinicians' subjective choice of regimen, which is not easily balanced via statistical methods. Nevertheless, univariate and multivariate analyses revealed no significant difference in pCR between the TCbHP and THP groups. Although the strength of our conclusion is limited by the small sample size, retrospective design, and baseline imbalances between the two groups, these results suggest that 6 cycles of the THP regimen may be a very effective dual-target neoadjuvant regimen for highly selected patient populations.

The common adverse events found in this study were neutropenia, anemia, thrombocytopenia, nausea, and diarrhea, although no patients experienced cardiac dysfunction. Similar results have been observed in previous studies (8-10). Interestingly, grade 3-4 adverse events were more common in the TCbHP group than in the THP group. Previous studies have indicated that EGFR is highly expressed on intestinal mucosal cells, and that pertuzumab inhibits the formation of a heterodimer between HER2 and
EGFR, which decreases the downstream activity of EGFR and leads to diarrhea. Carboplatin and docetaxel can also cause intestinal mucosal cell damage and can aggravate diarrhea when they are combined with pertuzumab $(18,19)$. The present study revealed that grade 3-4 diarrhea was significantly more common in the TCbHP group than in the THP group, which agrees with previously reported findings $(6,10,20)$. In the TRYPHAENA study, the incidences of grade $\geq 3$ thrombocytopenia were $11.8 \%$ for carboplatin-containing regimens and $0 \%$ for noncarboplatin-containing regimens (8). Similarly, the present study revealed that grade 3-4 thrombocytopenia was significantly more common in the TCbHP group than in the THP group. The KRISTINE study revealed carboplatin toxicity-related treatment interruption was required for $7.8 \%$ of patients $(17 / 219)$ in the TCbHP group, although the specific reactions were not described (10). The present study revealed very good cardiac safety in both groups, based on a small mean decrease in LVEF (4-5\%), which is similar to the result from the NeoSphere study. The THP group had a greater mean decrease in LVEF, which is likely related to the larger proportion of elderly patients, although no patients experienced serious cardiac toxicity.

In our study, the initial doses of chemotherapy drugs for a large number of patients did not follow those recommended in guidelines. Since our study is a retrospective study, it is not possible to obtain the specific reasons for the non-recommended dose. However, through the communication with the clinicians, it was found that the clinicians subjectively lowered the initial dose when setting the drug dose, taking into account the patient's age, obesity and underlying diseases. In the TCbHP group, carboplatin discontinuation (23.08\%) and dose reduction (53.85\%) were relatively common when the initial carboplatin dose was 5-6 $\mathrm{mg} / \mathrm{mL} / \mathrm{min}$. Interestingly, tolerance was significantly improved when the initial dose of carboplatin was 4$5 \mathrm{mg} / \mathrm{mL} / \mathrm{min}$, or even $3-4 \mathrm{mg} / \mathrm{mL} / \mathrm{min}$. These results suggest that Chinese patients do not have good tolerance of the TCbHP regimen, and potentially serious adverse reactions include grade 3-4 diarrhea and thrombocytopenia can occur. In these cases, reduction of the carboplatin dose and/or carboplatin discontinuation may be necessary. If chemotherapy is still intolerable after carboplatin discontinuation, the dose of docetaxel can be reduced, but the recommended dose should be maintained for the targeted drugs. Among our patients, the THP regimen was relatively well tolerated and serious adverse reactions were 
rare.

The present study has several limitations. Firstly, a small retrospective study has limited statistical power. Secondly, the small sample size might also be linked to selection bias. Finally, so far, almost all of our patients have not completed 1 year of adjuvant targeted therapy after surgery, and the relatively short follow-up period limited our ability to evaluate long-term survival and safety outcomes. Further studies with larger sample sizes and better methodology are needed to address these issues.

\section{Conclusions}

The TCbHP and THP regimens appear to be viable neoadjuvant treatments for Chinese patients with highrisk early or locally advanced HER2-positive breast cancer. Both regimens had generally good tolerability, although the TCbHP regimen had elevated incidences of grade 3-4 thrombocytopenia and diarrhea. For elderly patients who require neoadjuvant therapy, it appears that 6 cycles of the THP regimen is a neoadjuvant strategy with good efficacy and mild adverse events.

\section{Acknowledgments}

Funding: None.

\section{Footnote}

Reporting Checklist: The authors have completed the STROBE reporting checklist. Available at http://dx.doi. org/10.21037/gs-20-791

Data Sharing Statement: Available at http://dx.doi. org/10.21037/gs-20-791

Conflicts of Interest: All authors have completed the ICMJE uniform disclosure form (available at http://dx.doi. org/10.21037/gs-20-791). The authors have no conflicts of interest to declare.

Ethical Statement: The authors are accountable for all aspects of the work in ensuring that questions related to the accuracy or integrity of any part of the work are appropriately investigated and resolved. All procedures performed in this study involving human participants were in accordance with the Declaration of Helsinki (as revised in 2013). The study's retrospective protocol was approved by the ethics committee of The Affiliated Cancer Hospital of Zhengzhou University. All patients had provided informed consent for the research use of their data.

Open Access Statement: This is an Open Access article distributed in accordance with the Creative Commons Attribution-NonCommercial-NoDerivs 4.0 International License (CC BY-NC-ND 4.0), which permits the noncommercial replication and distribution of the article with the strict proviso that no changes or edits are made and the original work is properly cited (including links to both the formal publication through the relevant DOI and the license). See: https://creativecommons.org/licenses/by-nc-nd/4.0/.

\section{References}

1. Ross JS, Slodkowska EA, Symmans WF, et al. The HER2 receptor and breast cancer: ten years of targeted antiHER-2 therapy and personalized medicine. Oncologist 2009;14:320-68.

2. Yao M, Fu P. Advances in anti-HER2 therapy in metastatic breast cancer. Chin Clin Oncol 2018;7:27.

3. Brzezinska M, Dixon JM. Inflammatory breast cancer: no longer an absolute contraindication for breast conservation surgery following good response to neoadjuvant therapy. Gland Surg 2018;7:520-4.

4. Angelucci D, Tinari N, Grassadonia A, et al. Long-term outcome of neoadjuvant systemic therapy for locally advanced breast cancer in routine clinical practice. J Cancer Res Clin Oncol 2013;139:269-80.

5. Rastogi P, Anderson SJ, Bear HD, et al. Preoperative chemotherapy: updates of National Surgical Adjuvant Breast and Bowel Project Protocols B-18 and B-27. J Clin Oncol. 2008;26:778-85.

6. Gianni L, Pienkowski T, Im YH, et al. Efficacy and safety of neoadjuvant pertuzumab and trastuzumab in women with locally advanced, inflammatory, or early HER2-positive breast cancer (NeoSphere): a randomised multicentre, open-label, phase 2 trial. Lancet Oncol 2012;13:25-32.

7. Shao Z, Pang D, Yang H, et al. Efficacy, Safety, and Tolerability of Pertuzumab, Trastuzumab, and Docetaxel for Patients With Early or Locally Advanced ERBB2Positive Breast Cancer in Asia: The PEONY Phase 3 Randomized Clinical Trial. JAMA Oncol 2020;6:e193692.

8. Schneeweiss A, Chia S, Hickish T, et al. Longterm efficacy analysis of the randomised, phase II TRYPHAENA cardiac safety study: evaluating pertuzumab 
and trastuzumab plus standard neoadjuvant anthracyclinecontaining and anthracycline-free chemotherapy regimens in patients with HER2-positive early breast cancer. Eur J Cancer 2018;89:27-35.

9. van Ramshorst MS, van der Voort A, van Werkhoven ED, et al. Neoadjuvant chemotherapy with or without anthracyclines in the presence of dual HER2 blockade for HER2-positive breast cancer (TRAIN-2): a multicentre, open-label, randomised, phase 3 trial. Lancet Oncol 2018;19:1630-40.

10. Hurvitz SA, Martin M, Jung KH, et al. Neoadjuvant Trastuzumab Emtansine and Pertuzumab in Human Epidermal Growth Factor Receptor 2-Positive Breast Cancer: Three-Year Outcomes From the Phase III KRISTINE Study. J Clin Oncol 2019;37:2206-16.

11. Slamon D, Eiermann W, Robert N, et al. Adjuvant Trastuzumab in HER2-positive Breast Cancer. N Engl J Med 2011;365:1273-83.

12. Swain SM, Miles D, Kim SB, et al. Pertuzumab, trastuzumab, and docetaxel for HER2-positive metastatic breast cancer (CLEOPATRA): end-of-study results from a double-blind, randomised, placebo-controlled, phase 3 study. Lancet Oncol 2020;21:519-30.

13. von Minckwitz G, Huang CS, Mano MS, et al. Trastuzumab emtansine for residual HER2-positive breast cancer. N Engl J Med 2019;380:617-28.

14. Masuda N, Lee SJ, Ohtani S, et al. Adjuvant Capecitabine

Cite this article as: Lv M, Guo H, Wang C, Tian P, Ma Y, Chen X, Luo S. Neoadjuvant docetaxel with or without carboplatin plus dual HER2 blockade for HER2-positive breast cancer: a retrospective multi-center Chinese study. Gland Surg 2020;9(6):2079-2090. doi: 10.21037/gs-20-791 for Breast Cancer after Preoperative Chemotherapy. N Engl J Med 2017;376:2147-59.

15. von Minckwitz G, Procter M, de Azambuja E, et al. Adjuvant pertuzumab and trastuzumab in early HER2positive breast cancer. N Engl J Med 2017;377:122-31.

16. Seidman A, Hudis C, Pierri MK, et al. Cardiac dysfunction in the trastuzumab clinical trials experience. J Clin Oncol 2002;20:1215-21.

17. Mantarro S, Rossi M, Bonifazi M, et al. Risk of severe cardiotoxicity following treatment with trastuzumab: a meta-analysis of randomized and cohort studies of 29,000 women with breast cancer. Intern Emerg Med 2016;11:123-40.

18. Swain SM, Schneeweiss A, Gianni L, et al. Incidence and management of diarrhea in patients with HER2positive breast cancer treated with pertuzumab. Ann Oncol 2017;28:761-8.

19. Van Sebille YZ, Gibson RJ, Wardill HR, et al. ErbB small molecule tyrosine kinase inhibitor (TKI) induced diarrhoea: Chloride secretion as a mechanistic hypothesis. Cancer Treat Rev 2015;41:646-52.

20. Bines J, Procter M, Restuccia E, et al. Incidence and Management of Diarrhea With Adjuvant Pertuzumab and Trastuzumab in Patients With Human Epidermal Growth Factor Receptor 2-Positive Breast Cancer. Clin Breast Cancer 2020;20:174-181.e3. 